1 Bioprosthetic valve thrombosis and degeneration following transcatheter aortic

2 valve implantation (TAVI).

6 Bioprosthetic valve thrombosis (BPVT) is a recognised complication of prosthetic

7 aortic valves and can be seen in up to $13 \%$ of patients after transcatheter implantation.

8 The mechanism of BPVT is not well known, abnormal flow conditions in the new and

9 native sinuses and lack of functional endothelialisation are suspected causes. BPVT

10 may result in valve dysfunction, possibly related to degeneration, and recurrence of

11 patient symptoms, or remain subclinical. BPVT is best diagnosed by multiphase gated

12 CT angiography as the presence of reduced leaflet motion (RELM) and

13 hypoattenuating aortic leaflet thickening (HALT). Whilst CT is used to exclude

14 BPVT in symptomatic patients and those with increased valve gradient, the value of 15 screening and prophylactic anticoagulation is debatable.

Abbreviations:

BPVT: bioprosthetic valve thrombosis

19 BPHV: bioprosthetic heart valves

20 TTE: transthoracic echocardiography

21 TOE: transoesophageal echocardiography

22 CT: computed tomography

23 RELM: reduced leaflet motion

24 HALT: hypoattenuating aortic leaflet thickening

25 SVD: structural valve degeneration

\title{
Introduction:
}

Severe aortic stenosis is prevalent in the aging population with an incidence of $4.4 \%$ per year in patients $\geq 65$ years (1). Whilst surgical aortic valve replacement (SAVR) is the historical gold standard treatment in patients with symptomatic severe aortic stenosis, transcatheter aortic valve implantation (TAVI) initially provided a treatment option that is superior to medical therapy in inoperable and high-risk patients (2). 
More recent randomised trials indicated non-inferior or better short-medium term clinical outcomes of TAVI versus SAVR in intermediate-risk patients (3) and lowrisk patients (4). It is reported that each year, 180,000 patients could be considered potential TAVI candidates in the European Union and Northern-America, and this number might increase to 270,000 if the indications for TAVI were expanded to include low-risk patients (1). Whilst TAVI provides an alternative therapy to SAVR in inoperable and high-risk surgical patients with good short-medium term clinical outcomes, durable long-term outcome is important if the TAVI is to be offered to younger patients with lower surgical risk. Two key factors that may influence this is structural valve degeneration and leaflet thrombosis.

In this article we discuss the pathophysiology of bioprosthetic valve thrombosis (BPVT) and structural valve degeneration (SVD), their imaging findings, and the potential implications on clinical outcome.

\section{Bioprosthetic valve thrombosis}

BPVT is a recognised complication of surgical and transcatheter bioprosthetic heart valves (BPHV). The rate of BPVT varies significantly depending on the patient population, post-TAVI antithrombotic medication, diagnostic modality, and the definition of thrombosis. The incidence of BPVT is reported as $4 \%$ post-SAVR (5) and $13 \%$ post-TAVI (5). The incidence of clinically apparent BPVT thrombosis is much lower (0.6-2.8\%) (6,7) (Fig $1 \& 2)$, although significantly higher with valveinvalve TAVI (7.6\%) (8).

Reduced leaflet motion post-TAVI is more common in patients on dual antiplatelet therapy compared to those on warfarin (29\% vs. $0 \%$ ) (9). Thrombosis can occur within a few days after TAVI (10), although the reported median interval to BPVT in symptomatic patients is 181 days (interquartile range: $25-297$ days) (7) and remains significant at one year (11). It has been proposed that lack of anticoagulation may contribute to valve dysfunction, which may affect valve longevity (12). Registry data from 2,555 patients revealed a 5.5\% valve degeneration rate at 12 months, and anticoagulation was protective against valve degeneration (13). Whilst some studies have reported an association between subclinical leaflet thrombosis and transient ischaemic attack and stroke (5), others did not $(14,15)$. 


\section{Mechanism of BPVT}

The aetiology of TAVI thrombosis has not been fully elucidated, but likely to be multifactorial, with contribution from the components of Virchow's triad (16). These are likely to be (i) device-related, (ii) rheological, (iii) haematological and (iv) patientrelated factors.

\section{(i) Device-related factors}

Implantation of artificial surfaces such as a prosthetic valve that is primarily composed of collagen, which comes into contact with flowing blood results in contact activation of plasma coagulation. Proteins such as von Willebrand factor, fibrinogen, and fibronectin are adsorbed onto the artificial surface and bring about accumulation of components of the contact system on the valve (17), followed by adhesion of platelets and leucocytes, platelet activation and aggregation, and thrombin generation. This can eventually result in deposition of a platelet-rich thrombus held together by fibrin strands on the artificial valve surface. Endothelialisation of the bioprosthetic valve usually occurs around 3 months post-implant (18), but valve thrombosis continues to occur well beyond this time-window. The stented native valve itself may contribute to an ongoing thrombotic risk, since in patients with severe aortic stenosis, raised levels of tissue factor and activated factor XI have been documented, correlating with the degree of measurable thrombin generation in venous blood (19-21). The TAVI implantation procedure may also increase the risk of valve thrombosis. Crimping of the bioprosthetic valve leaflets into the delivery system, incomplete expansion, post-dilatation and incomplete apposition to the aortic wall have all been proposed as possible contributors to subsequent BPVT (22-24).

\section{(iii) Rheological factors}

Stagnant or static flow results in activation of coagulation, whilst turbulent flow can lead to platelet activation and neointimal trauma and disruption, and may increase the likelihood of local thrombus formation. Incomplete expansion or apposition of the prosthesis to the native aortic valve may produce complex localised flow patterns which promote fibrin deposition, platelet activation and thrombosis $(22,25)$.

High wall shear stress from the base of the valve leaflets toward the leaflet tips, where maximum flow activation occurs can promote platelet activation $(26,27)$. Prolonged 
exposure of blood to these shear stresses (28-29) combined with flow recirculation may stimulate localised thrombogenesis (26).

The area between the prosthetic valve frame and the stented native valve leaflets is also recognised to be an area of low shear but with potential turbulent flow pattern which can create a prothrombotic milieu $(6,27)$. It has also been proposed that supravalvular, rather than intra-annular, positioning of the prosthetic valve may reduce the risk of valve leaflet thrombosis (27).

\section{(iv) Haematological factors}

Regardless of the trigger (such as rheological factors or contact activation), the end result is activation of both platelet aggregation and the coagulation cascade. However, intrinsic defects in the coagulation pathways that promote a hypercoagulable state may also predispose to valve thrombosis. Procoagulant conditions such as inherited or acquired thrombophilias all increase the predisposition to thrombotic events (30). A testament to the crucial role of the coagulation pathway in valve leaflet thrombosis is that the thrombosis resolves with therapeutic anticoagulation and anticoagulation is also known to reduce restricted leaflet motion (31).

\section{(v) Patient-related factors}

The majority of TAVI recipients are elderly, often with multiple comorbidities that increase the risk of thrombosis after TAVI. Patient-related factors that increase the propensity for thrombosis include atrial fibrillation (AF), age, immobility, malignancy, smoking and renal failure (32), with AF being perhaps the most recognised risk factor. Most patients undergoing TAVI have a $\mathrm{CHADS}_{2} \mathrm{VASC}_{2} \mathrm{score}$ of at least 3 (usually 1-2 for age, usually accompanied by other vascular arterial disease or hypertension, which are extremely common in these patients). AF is frequently observed in this cohort, even when not previously reported (33), and underscores the need for a low index of suspicion for AF in this cohort, where new onset $\mathrm{AF}$ is reported in $14 \%$ post-TAVI (33).

\section{Mechanisms of structural valve degeneration (SVD)}

SVD is an acquired disease of the bioprosthetic valves and is defined as deterioration of the leaflets (eg. thickening, calcification, tearing) or supporting structures. These would adversely affect the valve hemodynamic and manifest in valular stenosis and/or 
regurgitation (34).There is a continued interest to delve further into our understanding of the biology and mechanisms of SVD and BVF. In particular, this seems of importance in the context of the increasing focus on the treatment of valvular disease and increasingly in younger patients. From an imaging and radiological perspective, understanding of SVD mechanisms is imperative to guide the development of new imaging approaches to improve patient outcomes.

\section{(i) Leaflet thrombus, fibrosis, and calcification}

The patho-anatomical characteristics of SVD is determined from studies of explanted valves and the association of SVD with circulating biomarkers. On gross inspection, longer-term explanted valves frequently demonstrate leaflet tears, pannus or fibrosis, and/or calcification (Fig.3; A\&B), which lead to the restricted leaflet motion or insufficiency that can be observed on imaging the valve. The timing of leaflet thickening secondary to fibrosis and calcification in transcatheter explants was recently reported at greater than 60 days and greater than 4 years, respectively (Fig. 3; C\&D) (35-36). However, an equivalent timeline of histological fibrosis and calcification across the many types of surgical bioprosthesis used since the 1960s is unclear. Notably, these timelines have the potential for bias given the 'snapshot in time' nature of explanted tissue. Long-term follow-up imaging studies have the potential for elucidating this in the future.

Studies in both surgical and transcatheter heart valves demonstrate that calcification can severely disrupt leaflet structure and function. Mineralisation is proposed to be the result of a combination of factors including biological response to matrix fixative materials (eg. glutaraldehyde), mechanical stress, and cellular infiltration (37-39). In fact, studies have demonstrated an association of surgical BPHV calcification and activation of pathways traditionally identified in vascular calcification, including expression of alkaline phosphatase and osteopontin (40-42). In addition to providing insights into fibrosis and calcification, studies of explanted transcatheter bioprosthetic valves reported histologically observable thrombus. However, rates varied considerably, which may be attributable to different histological methods and diagnostic criteria used in each study $(35,36)$. Also reported was the predominate accumulation of thrombus at the base of leaflets on explanted transcatheter valves, as seen on computed tomography (CT) imaging. Yet establishing what is equivalent of leaflet thrombus histologically and the appearance of 
hypoattenuating leaflet thickening (HALT) on imaging of either surgical or transcatheter heart valves remains to be seen $(31,35,43)$. There is also still a need to establish if there is a direct molecular mechanism of leaflet thrombus as a driver of SVD. Importantly, future studies, such as the planned long-term durability analysis of the CT sub-study of PARTNER3 (44) will help to clarify any potential link between SVD and leaflet thrombus.

\section{(ii) Cholesterol and inflammation}

The presence of cholesterol in surgical bioprosthetic valves has been reported over many years in the explanted valves and patients with SVD have higher cholesterol levels (45-49). Oxidised low density lipoprotein (LDL) has also been found in degenerated BPHVs and is associated with inflammatory infiltrates. Moreover, blood levels of LDL and lipoprotein-associated phospholipase A2 have been found to be an independent predictor of SVD in SAVR patients $(46,50,51)$. While these studies propose a potential metabolic link to SVD, identifying specific risk factors or markers of SVD requires further study. Similarly, the debate on statin use to combat SVD remains, with conflicting results reported. Integration of molecular imaging techniques may allow for the evaluation of lipid and inflammatory infiltration to bioprosthetic valves akin to current atherosclerotic plaque approaches. Recently, Cartlidge et al. demonstrated that 18F-Fluoride uptake was associated with areas of SVD on histology on ex-vivo surgical BPHVs (52). However, further refinement of our understanding of the cellular and matrix processes being detected by $18 \mathrm{~F}$-Fluoride imaging is needed. The continued evolution of imaging and computing technologies also hold promise for understanding the pathophysiology of SVD. This includes applications of radiomics as well as computational fluid dynamics to understanding flow characteristics and shear stresses around the bioprosthetic valve to understand the causes of BPVT and degeneration.

\section{Diagnosis of bioprosthetic valve thrombosis and degeneration}

Valve thrombosis should be considererd as a clinical and imaging spectrum, from subclinical, non-obstructive thrombosis to clinical-obstructive thrombosis (Fig. 1). The development of obstructive symptoms are dependent on the volume of thrombus, the number of leaflets involved, and the length of time from implant (9). Based on this 
hypothetical concept, the echocardiographic signs of valve thrombosis are diverse, ranging from a totally normal appearance to complete valvular dysfunction.

205 Transthoracic echocardiography (TTE) is the primary imaging modality during

206 follow-up for for monitoring prosthetic valve function (Table 1; Fig. 4\&5).

207 European guidelines recommend baseline TTE evaluation within 30 days of

208 implantation, at 1 year, and then annually afterwards. Earlier follow up studies should

209 be considered when new symptoms occur (53). First-line screening of prosthetic valve 210 dysfunction with TTE has limited value, as gradients could be normal despite valve

211 thrombosis (32). BPVT should be considered when mean trans-prosthetic pressure 212 gradient ( $\mathrm{mPG}$ ) is $\geq 20 \mathrm{mmHg}$, or when a $>50 \%$ increase in $\mathrm{mPG}$ from baseline is 213 observed (54-56) (Table 1). Leaflet thickening may be visualised (Fig. 4) as the 214 echocardiographic correlate of HALT on CT (57). However, acoustic shadowing from 215 the TAVI device may preclude adequate visualisation of the TAVI device leaflets (6) 216 and transvalvular gradients depend on the type and size of the implanted valve, so 217 unless there is a significant change from baseline gradients, TTE may not be sensitive 218 enough to detect early haemodynamic changes in BPVT. Transoesophageal 219 echocardiography (TOE) is reported to have comparable sensitivity to CT for the 220 detection of leaflet thickening, thrombotic appositions, or restricted leaflet mobility 221 (9), and though more invasive than TTE or CT, it could be considered when TTE 222 images are suboptimal and in patients at increased risk of iodine-induced 223 nephropathy. A deeper TOE longitudinal view with slight anterior flexion of the 224 probe is recommended to avoid acoustic shadows created by the bioprosthesis 225 scaffold. ECG gated CT angiography is recognised as the gold standard imaging technique for 228 the diagnosis of BPVT. CT diagnosis is based on the observation of reduced leaflet 229 motion (RELM) and HALT on multiphase ECG gated CT angiography $(9,12,58)$ (Fig $2302 \& 6$ ). The leaflet thickening commences from the base of the leaflet and extends to 231 the tip in more severe cases. Based on the extent of leaflet thickening (eg. basal vs. 232 whole leaflet involvement), a semi-quantitative HALT grading system has been 233 described (43). Leaflet thickening and/or calcification are observed in cases with 234 leaflet degeneration (Fig. 7). Previous studies indicated that CT attenuation can be 235 measured to differentiate pannus from thrombus ( $\geq 145 \mathrm{HU}$ and $<90 \mathrm{HU}$, respectively) 236 (59). It should be recognised that pannus and thrombus can coexist (60). When 
leaflets are not thickened or restricted, a prosthesis-patient mismatch should be considered as the cause of symptoms or abnormal transvalvular gradients (Fig. 7).

240 Leaflet motion and opening are evaluated on systolic phase images, and leaflet 241 thickening may be observed in both systolic or diastolic images (Fig. 6). To reduce 242 beam hardening artefacts from TAVI struts, CT is performed with a tube voltage of $243120-140 \mathrm{kV}$, the tube current is adjusted based on the patient's body morphology.

244 Beam hardening is less of an issue with stented and stentless SAVR and imaging at $245100 \mathrm{kV}$ is feasible. Unless contraindicated, heart rate control strategies (eg. beta-

246 blockade and avoiding caffeine prior to the scan) should be considered to improve the 247 visualisation of bioprosthetic valve leaflets.

248 Whilst retrospective ECG gating was recommended by previous studies (61), 249 prospective gating with wide padding (eg. 30-90\%) may be considered to reduce 250 radiation exposure. The imaging field can be limited to the TAVI structure and the 251 left ventricular outflow tract (Fig. 8) to reduce radiation burden. Images are reconstructed with iterative reconstruction and thin slices $(0.5-0.625 \mathrm{~mm})$ at $5-10 \%$ intervals. When available, ECG gated dual-energy imaging may be helpful to reduce beam hardening from the valve struts and improve visualisation of the leaflets.

\section{Management of bioprosthetic valve thrombosis}

257 There is no universal approach for the prevention of BPVT and/or routine imaging screening of BPVT (7). Routine anticoagulation is associated with increased bleeding risk, particularly in an elderly population, particularly intracranial haemorrhage (62).

260 Current multisociety guidelines recommend administration of aspirin and 3 to 6

261 months of concomitant clopidogrel or vitamin K antagonist alone (63).

262 Whilst the risk of BPVT is significantly lower with oral anticoagulation, the 263 protective effectiveness of dual antiplatelet therapy is questionable $(5,7)$. The routine 264 application of non-vitamin $\mathrm{K}$ antagonist oral anticoagulant therapies for the prevention of BPVT had mixed results: one major trial (GALILEO trial) comparing the efficacy of rivaroxaban with antiplatelet regiments was prematurely terminated due to safety concerns related to increased all-cause death, thromboembolic events, and bleeding in the intervention arm (64). Another major trial (ATLANTIS) is

269 ongoing (65). Preventive anticoagulation may be considered in patients at higher risk 270 of BPVT (e.g. co-existing AF, valve-in-valve procedure) $(7,56)$. BPVT is reported to 
271 resolve in $88 \%$ of cases within 2 months of anticoagulation (6). The reported median

272 time to the reduction of transvalvular gradients is 14 days (7). In some patients, only

273 partial reduction in valve gradient and thrombus load may be seen due to the

274 organisation of the clot (7) and/or leaflet fibrosis.

275

276 The routine application of follow-up CT imaging at specified intervals is not currently

277 recommended (43). CT or TOE should be considered in patients with recurrent

278 symptoms (exertional dyspnea and stroke), increased gradients or new transvalvular

279 regurgitation.

280

\section{Conclusion:}

282 Bioprosthetic valve thrombosis is a complication of the TAVI procedure. BPVT may

283 result in the recurrence of symptoms in only a subset of patients, depending on

284 thrombus load. The emerging evidence indicates that thrombosis may lead to valve

285 degeneration. Imaging investigations play a fundamental role in the diagnosis of

286 BPVT and should be considered in suspected cases. Increased awareness of this

287 potential complication, including its potential impact on longer term valve function

288 and stroke risk, together with future development of imaging and haematological

289 biomarkers may help earlier identification of high risk patients and those with

290 subclinical BPVT that would benefit from anticoagulation. 


\section{References:}

1. Cahill TJ, Chen M, Hayashida K, et al. Transcatheter aortic valve implantation: current status and future perspectives. Eur Heart J. 2018 Jul 21;39(28):2625-2634.

2. Leon MB, Smith CR, Mack M, et al. Transcatheter aortic-valve implantation for aortic stenosis in patients who cannot undergo surgery. N Engl J Med. 2010 Oct 21;363(17):1597-607.

3. Leon MB, Smith CR, Mack MJ, et al. Transcatheter or Surgical Aortic-Valve Replacement in Intermediate-Risk Patients. N Engl J Med. 2016 Apr 28;374(17):1609-20.

4. Mack MJ, Leon MB, Thourani VH, et al. Transcatheter aortic-valve replacement with a balloon-expandable valve in low-risk patients (PARTNER 3 Trial). NEJM, March 17, 2019, at NEJM.org

5. Chakravarty T, Søndergaard L, Friedman J, et al. Subclinical leaflet thrombosis in surgical and transcatheter bioprosthetic aortic valves: an observational study. Lancet. 2017 Jun 17;389(10087):2383-2392.

6. Latib A, Naganuma T, Abdel-Wahab M, et al. Latib A, et al. Treatment and clinical outcomes of transcatheter heart valve thrombosis. Circ Cardiovasc Interv. 2015 Apr;8(4).

7. Jose J, Sulimov DS2, E-Mawardy M, et al. Clinical Bioprosthetic Heart Valve Thrombosis After Transcatheter Aortic Valve Replacement: Incidence, Characteristics, and Treatment Outcomes. JACC Cardiovasc Interv. 2017 Apr 10;10(7):686-697. doi: 10.1016/j.jcin.2017.01.045.

8. Abdel-Wahab M, Simonato M, Latib A, et al. Clinical Valve Thrombosis After Transcatheter Aortic Valve-in-Valve Implantation. Circ Cardiovasc Interv. 2018 Nov;11(11):e006730. doi: 10.1161/CIRCINTERVENTIONS.118.006730.

9. Makkar RR, Fontana G, Jilaihawi H, et al. Possible subclinical leaflet thrombosis in bio- prosthetic aortic valves. N Engl J Med 2015;373: 2015-24.

10. Pache G, Schoechlin S, Blanke P, et al. Early hypo-attenuated leaflet thickening in balloon-expandable transcatheter aortic heart valves. Eur Heart J 2016; 37: 2263-71.

11. Yanagisawa R, Hayashida K, Yamada Y, et al. Incidence, Predictors, and MidTerm Outcomes of Possible Leaflet Thrombosis after TAVR. JACC Cardiovasc Imaging 2016;10:1-11.

12. Del Trigo M, Munoz-Garcia AJ, Wijeysundera $\mathrm{HC}$ et al. Incidence, Timing, and Predictors of Valve Hemodynamic Deterioration After Transcatheter Aortic Valve Replacement: Multicenter Registry. Journal of the American College of Cardiology 2016;67:644-55.

13. Overtchouk P., Guedeney P., Rouanet S., et al. Long-Term Mortality and Early Valve Dysfunction According to Anticoagulation Use. J Am Coll Cardiol 2019;73(1):13-21. 
14. Yanagisawa R., Tanaka M., Yashima F., et al. Early and Late Leaflet Thrombosis After Transcatheter Aortic Valve Replacement. Circ Cardiovasc Interv 2019;12(2).

15. Kalra A., Raza S., Puri R., et al. Subclinical Leaflet Thrombosis and Clinical Outcomes after TAVR: A Systematic Review and Meta-Analysis. Struct Hear 2018;2(3):223-8.

16. Dangas GD, Weitz JI, Giustino G, Makkar R, Mehran R. Prosthetic Heart Valve Thrombosis. J Am Coll Cardiol 2016;68:2670 -89.doi:10.1016/j.jacc.2016.09.958.

17. Farndale RW, Sixma JJ, Barnes MJ, de Groot PG. The role of collagen in thrombosis and hemostasis. J Thromb Haemost. 2004 Apr;2(4):561-73.

18. Noble S, Asgar A, Cartier R, Virmani R, Bonan R. Anatomo-pathological analysis after CoreValve revalving system implantation. Eurointervention. (2009) 78-85. doi: 10.4244/EIJV5I1A12.

19. Luszczak J, Undas A, Gissel M, Olszowska M, Butenas S. Activated factor XI and tissue factor in aortic stenosis: links with thrombin generation.Blood Coagul Fibrinolysis. 2011; 22:473-479. doi: 10.1097/MBC.0b013e328346c2bb

20. Breyne J, Juthier F, Corseaux D, et al. Atherosclerotic-like process in aortic stenosis: activation of the tissue factor-thrombin pathway and potential role through osteopontin alteration. Atherosclerosis 2010;213:369-376.

21. Marechaux S, Corseaux D, Vincentelli A, et al. Identification of tissue factor in experimental aortic valve sclerosis. Cardiovasc Pathol 2009;18:67-76.

22. Mylotte D, Andalib A, Thériault-Lauzier P, et al. Transcatheter heart valve failure: a systematic review. Eur Heart J 2015;36:1306-27.

23. Fuchs A, De Backer O, Brooks M, et al. Subclinical leaflet thickening and stent frame geometry in self- expanding transcatheter heart valves. Eurointervention. (2017) 9:e1067-75. doi: 10.4244/EIJ-D-17-00373.

24. Alavi SH, Groves EM, Kheradvar A. The effects of transcatheter valve crimping on pericardial leaflets. Ann Thorac Surg. 2014 Apr;97(4):1260-6. doi: 10.1016/j.athoracsur.2013.11.009.

25. Yoganathan AP He Z, Casey Jones S. Fluid mechanics of heart valves. Annu Rev Biomed Eng 2004;6:33162.doi:10.1146/annurev.bioeng.6.040803.140111.

26. Kopanidis A, Pantos I, Alexopoulos N, Theodorakakos A, Efstathopoulos E, Katritsis D. Aortic flow patterns after simulated implantation of transcatheter aortic valves. Hellenic J Cardiol 2015;56:418-28.

27. Vahidkhah K, Barakat M, Abbasi M, et al. Valve thrombosis following transcatheter aortic valve replacement: significance of blood stasis on the leaflets. Eur J Cardiothorac Surg. 2017;51;927-35.

28. Bluestein D, Rambod E, Gharib M. Vortex shedding as a mechanism for free emboli formation in mechanical heart valves. J Biomech Eng. 2000; 122: 125134. 
29. Ducci A, Tzamtzis S, Mullen MJ, Burriesci G. Hemodynamics in the Valsalva sinuses after transcatheter aortic valve implantation (TAVI). J Heart Valve Dis. 2013 Sep;22(5):688-96.

30. Gencbay M, Turan F, Degertekin M, Eksi N, Mutlu B, Unalp A. High prevalence of hypercoagulable states in patients with recurrent thrombosis of mechanical heart valves. J Heart Valve Dis 1998;7:601-9.

31. Sondergaard L, De Backer O, Kofoed KF et al. Natural history of subclinical leaflet thrombosis affecting motion in bioprosthetic aortic valves. European heart journal 2017;38:2201-2207.

32. Rosseel L, De Backer O, Søndergaard L. Clinical Valve Thrombosis and Subclinical Leaflet Thrombosis Following Transcatheter Aortic Valve Replacement: Is There a Need for a Patient-Tailored Antithrombotic Therapy? Front. Cardiovasc. Med. 2019;6:44. doi: 10.3389/fcvm.2019.

33. Kalra R, Patel N, Doshi R, Arora G, Arora P. JAMA Intern Med. 2019;179(8):1122-1130. doi:10.1001/jamainternmed.2019.0205.

34. Dvir D, Bourguignon T, Otto CM, et al. Standardized Definition of Structural Valve Degeneration for Surgical and Transcatheter Bioprosthetic Aortic Valves.Circulation. 2018 Jan 23;137(4):388-399. doi: 10.1161/CIRCULATIONAHA.117.030729.

35. Sellers SL, Turner CT, Sathananthan J et al. Transcatheter Aortic Heart Valves: Histological Analysis Providing Insight to Leaflet Thickening and Structural Valve Degeneration. JACC Cardiovasc Imaging. 2019 Jan;12(1):135-145. doi: 10.1016/j.jcmg.2018.06.028

36. Yahagi K, Ladich E, Kutys R et al. Pathology of balloon-expandable transcatheter aortic valves. Catheter Cardiovasc Interv. 2017; 15;90(6):10481057. doi: $10.1002 / \mathrm{ccd} .27160$

37. Chen W, Schoen FJ, Levy RJ. Mechanism of efficacy of 2-amino oleic acid for inhibition of calcification of glutaraldehyde-pretreated porcine bioprosthetic heart valves. Circulation 1994;90:323-9.

38. Pibarot P, Dumesnil JG. Prosthetic heart valves: selection of the optimal prosthesis and long-term management. Circulation 2009;119:1034-48.

39. Rodriguez-Gabella T, Voisine P, Puri R, Pibarot P, Rodes-Cabau J. Aortic Bioprosthetic Valve Durability: Incidence, Mechanisms, Predictors, and Management of Surgical and Transcatheter Valve Degeneration. Journal of the American College of Cardiology 2017;70:1013-1028.

40. Srivatsa SS, Harrity PJ, Maercklein PB et al. Increased cellular expression of matrix proteins that regulate mineralization is associated with calcification of native human and porcine xenograft bioprosthetic heart valves. The Journal of clinical investigation 1997;99:996-1009.

41. Lu F, Wu H, Bai Y et al. Evidence of Osteogenic Regulation in Calcific Porcine Aortic Valves. The heart surgery forum 2018;21:E375-e381.

42. Shen M, Marie P, Farge D et al. Osteopontin is associated with bioprosthetic heart valve calcification in humans. Comptes rendus de l'Academie des sciences Serie III, Sciences de la vie 1997;320:49-57. 
43. Blanke P, Weir-McCall JR, Achenbach S et al. Computed tomography imaging in the context of transcatheter aortic valve implantation (TAVI) / transcatheter aortic valve replacement (TAVR): An expert consensus document of the Society of Cardiovascular Computed
cardiovascular computed tomography 2019;13:1-20.

44. Makkar RR. PARTNER 3 low-risk computed tomography sub-study: subclinical leaflet thrombosis in transcatheter and surgical bioprosthetic valves. Presented at: TCT 2019. September 27, 2019. San Francisco CA.

45. Ferrans VJ, McManus B, Roberts WC. Cholesteryl ester crystals in a porcine aortic valvular bioprosthesis implanted for eight years. Chest 1983;83:698701.

46. Shetty R, Girerd N, Cote N et al. Elevated proportion of small, dense lowdensity lipoprotein particles and lower adiponectin blood levels predict early structural valve degeneration of bioprostheses. Cardiology 2012;121:20-6.

47. Farivar RS, Cohn LH. Hypercholesterolemia is a risk factor for bioprosthetic valve calcification and explantation. The Journal of thoracic and cardiovascular surgery 2003;126:969-75.

48. Nsaibia MJ, Mahmut A, Mahjoub $\mathrm{H}$ et al. Association between plasma lipoprotein levels and bioprosthetic valve structural degeneration. Heart (British Cardiac Society) 2016;102:1915-1921.

49. Mahjoub H, Mathieu P, Senechal M et al. ApoB/ApoA-I ratio is associated with increased risk of bioprosthetic valve degeneration. Journal of the American College of Cardiology 2013;61:752-61.

50. Shetty R, Pibarot P, Audet A et al. Lipid-mediated inflammation and degeneration of bioprosthetic heart valves. European journal of clinical investigation 2009;39:471-80.

51. Mahmut A, Mahjoub H, Boulanger MC et al. Lp-PLA2 is associated with structural valve degeneration of bioprostheses. European journal of clinical investigation 2014;44:136-45.

52. Cartlidge T, White A, Van Beek E, Newby D, Dweck M. 18F-fluoride positron emission tomography: computed tomography angiography predicts bioprosthetic valve degeneration. Journal of the American College of Cardiology 2018;71:A1452.

53. Baumgartner H, Falk V, Bax JJ, De Bonis M, Hamm C, Holm PJ, et al. 2017ESC/EACTS Guidelines for the management of valvular heart disease. The task force for the management of valvular heart disease of the European society of cardiology (ESC) and the European association for cardio-thoracic surgery (EACTS). Eur H J. (2017) 38:2739-91. doi: 10.1093/eurheartj/ehx391

54. Hahn RT, Little SH, Monaghan MJ, et al. Recommendations for comprehensive intraprocedural echocardio- graphic imaging during TAVR. JACC Cardiovasc Imaging 2015; 8:261-87.

55. Puri R, Auffret V, Rodés-Cabau J. Bioprosthetic valve thrombosis. J Am Coll Cardiol. 2017;69(17):2193-211. 
56. Egbe AC, Pislaru SV, Pellikka PA, et al. Bioprosthetic valve thrombosis versus structural failure: clinical and echocardiographic predictors. J Am Coll Cardiol 2015; 66:2285-94.

57. Hamadanchi A, Bäz L, Möbius-Winkler S, et al. Basal leaflet thickening and color paucity in the echocardiographic evaluation of subclinical leaflet thrombosis after transcatheter aortic valve replacement Int J Cardiovasc Imaging (2019) 35: 119. https://doi.org/10.1007/s10554-018-1439-5.

58. Hansson NC, Grove EL, Andersen HR. Transcatheter aortic valve thrombosis: incidence, predisposing factors, and clinical implications. J Am Coll Cardiol 2016; 68: 2059-69.

59. Gündüz S1, Özkan M, Kalçik M, et al. Sixty-Four-Section Cardiac Computed Tomography in Mechanical Prosthetic Heart Valve Dysfunction: Thrombus or Pannus. Circ Cardiovasc Imaging. 2015 Dec;8(12). pii: e003246. doi: 10.1161/CIRCIMAGING.115.003246.

60. Vitale N, Renzulli A, Agozzino L, et al. Obstruction of mechanical mitral prostheses: analysis of pathologic findings. Ann Thorac Surg. 1997;63:11011106.

61. Midha PA, Vrishank Raghav, Rahul Sharma, et al. The Fluid Mechanics of Transcatheter Heart Valve Leaflet Thrombosis in the Neosinus. Circulation. 2017 Oct 24;136(17):1598-1609.

62. Fang MC, Go AS, Hylek EM, et al. Jensvold, and Daniel E. Singer. Age and the Risk of Warfarin-Associated Hemorrhage: The Anticoagulation and Risk Factors In Atrial Fibrillation Study. J Am Geriatr Soc. 2006 Aug; 54(8): 12311236.

63. Holmes DR Jr., Mack MJ, Kaul S, et al. 2012 ACCF/AATS/SCAI/STS expert consensus document on transcatheter aortic valve replacement. J Am Coll Cardiol 2012;59:1200-54.

64. De Backer O, Dangas GD, Jilaihawi H, et al. Reduced Leaflet Motion after Transcatheter Aortic-Valve Replacement. N Engl J Med. 2019 Nov 16. doi: 10.1056/NEJMoa1911426.

65. Collet JP, Berti S, Cequier A, Van Belle E, Lefevre T, Leprince P, Neumann FJ, Vicaut E, Montalescot G. Oral anti-Xa anticoagulation after trans-aortic valve implantation for aortic stenosis: The randomized ATLANTIS trial. Am Heart J. 2018 Jun;200:44-50.

66. Marcoff L, Koulogiannis KP, Aldaia L, Fuschetto DP, Kipperman RM, Laighold S, Gillam LD. Color Paucity as a Marker of Transcatheter Valve Thrombosis. JACC Cardiovasc Imaging. 2017;10(1):78-81. 
504

505

506

507

508

509

510

511

512

513

514

515

516

517

\section{Table legends:}

Table 1: Echocardiographic (TTE/TOE) signs of aortic bioprosthetic valve thrombosis.

\section{Figure legends:}

Fig. 1. The clinical and imaging spectrum of bioprosthetic valve thrombosis (BPVT).

In the early stages of BPVT, the patients may not be symptomatic or have significant transvalvular gradients on echocardiography. An increase in leaflet thrombus load and/or leaflet fibrosis results in valve restriction, increased transvalvular gradients, and recurrence of symptoms. Physicians should be aware that the symptoms and gradient changes may initially be mild, and early identification would aid appropriate earlier management.

Fig 2. Sub-clinical TAVI thrombosis.

81 years old patient referred for a CT scan to investigate the cause of hoarse voice and weight loss, 45 days post TAVI. Ungated contrast-enhanced CT demonstrated no cause for the presenting symptoms. The incidental finding was hypoattenuating leaflet thickening indicating thrombosis (HALT; A\&B). Transthoracic echocardiogram (performed prior to $\mathrm{CT}$ ) demonstrated a mean trans-prosthetic gradient of $10 \mathrm{mmHg}$ that reduced to $2 \mathrm{mmHg}$ following anticoagulation. The patient was treated with nonvitamin $\mathrm{K}$ antagonist oral anticoagulant. A follow up CT demonstrated resolution of the thrombi, 4 months following treatment (C\&D).

Fig 3. Examples of features of bioprosthetic aortic valve pathology seen on gross examination at explant and on histology. A\&B: surgical BPHV explants demonstrating grossly observable fibrosis and calcification on the aortic aspect of pericardial leaflets. C. Leaflet thickening as the result of fibrosis on an explanted 
transcatheter aortic valve leaflet seen on histological cross-section staining with

533 Movat's Pentachrome stain. D. Severe calcification of an explanted aortic

534 transcatheter BPHV pericardial leaflet shown on histological section stained with

535 Movat's pentachrome.

536 Fig 4. Bioprosthetic leaflet thickening on echocardiogram and CT in a patient with

537 transcatheter aortic valve thrombosis.

538 Modified trans-oesophageal long axis echocardiographic view of the bioprosthetic

539 aortic valve shows wedge-shaped thickening of the valve leaflet (yellow arrow) with

540 correlating CT image (red arrow; B). Follow up imaging 3 months after

541 anticoagulation therapy documents resolution of basal leaflet thickening (C).

543 Fig 5. Colour paucity sign on echocardiogram in a patient with bioprosthetic valve 544 thrombosis.

545 80-year-old man presented with transient ischaemic attack 2 months after TAVI.

546 Transoesophageal echocardiogram showed leaflet thickening (arrow, A) and the

547 "colour paucity" sign, a filling defect in colour flow caused by the thrombus (black

548 arrows, B). A peak trans-prosthetic velocity of $>4 \mathrm{~m} / \mathrm{s}$ indicates severe stenosis (C).

549 3D echo images (D) demonstrated thrombi on valve leaflets (open arrow).

551 Fig 6. Bioprosthetic aortic valve thrombosis on CT.

55282 years old patient presenting with high trans-prosthetic gradients on

553 echocardiogram, 4months after transcatheter aortic valve implantation $(27 \mathrm{~mm}$ Lotus,

554 Boston Scientific). The leaflets were not visible on transthoracic echocardiogram

555 (TTE). CT demonstrated moderate hypoattenuating leaflet thickening (HALT)

556 extending to the leaflet tips (A). Mid systolic images (B) demonstrated reduced leaflet 
motion (RELM). Follow up CT 6 months after oral anticoagulation showed partial resolution of the HALT (C) and improved valve opening (D). Follow up TTE confirmed reduction of the mean trans-prosthetic gradient from $28 \mathrm{mmHg}$ to

$56010 \mathrm{mmHg}$.

562 Fig 7. The spectrum of valve disease in patients with increased trans-prosthetic 563 gradients.

564 Valve thrombosis demonstrated on axial and coronal multiplanar CT reformats (A:

565 reduced leaflet motion; B: Hypoattenuating leaflet thickening). Degenerated

566 transcatheter bioprosthetic leaflet calcification is seen in a patient with previous

567 transcatheter aortic valve implantation within a calcified homograft (C \& D). Image D

$568 \&$ E shows an under-deployed transcatheter aortic valve (34mm Medtronic Evolut R;

569 Minneapolis, MN) as a result of heavy native valve calcification with resultant small

570 valve area for patient's size. The mean trans-prosthetic gradient was $32 \mathrm{mmHg}$

571 following implantation of the valve (E\&F).

572

573 Fig 8. CT imaging of bioprosthesis.

574 Scout image (A) is used to identify the bioprosthesis (arrow) and plan limited field of

575 view (yellow box) CT angiogram ( $8 \mathrm{~cm}$ coverage in $\mathrm{z}$ axis). The resultant dose length 576 product (DLP) was $175 \mathrm{mG} . \mathrm{cm}$. 